\title{
Prevalence and Predictors of Use of Herbal Medicines Among The Most Recently Delivered Mothers in Tabora Municipality, Tanzania
}

Anna Tengia Kessy ( $\square$ atengia@gmail.com )

Muhimbili University of Health and Allied Sciences https://orcid.org/0000-0003-2942-7959

George Chombe Msalale

Kitete Regional Hospital

\section{Research}

Keywords: Herbal use, pregnancy, recently delivered, Tabora, Tanzania

Posted Date: May 6th, 2020

DOI: https://doi.org/10.21203/rs.3.rs-25402/v1

License: (c) (i) This work is licensed under a Creative Commons Attribution 4.0 International License.

Read Full License 


\section{Abstract}

Background In most of sub-Sahara African countries, herbal medicines are widely used during pregnancy and labor for various motives despite their unclear pharmacology and potential toxicity. Considering the Sustainable Development Goals, exposure to herbs during pregnancy should be restricted to safeguard the health of mothers and newborns. Thus, this study aims to assess the proportion of mothers using herbal medicines during pregnancy and delivery and to determine factors associated with the practice.

Methods We used an interview schedule to gather information among 340 mothers who delivered a live baby in the preceding two years. Using a two-stage-sampling technique, we selected and interviewed mothers at the health facilities. We compared proportions using chi-square test. We performed a Poisson logistic regression analysis to determine independent predictors of herbal use.

Results Over $60 \%$ of mothers in Tabora municipality used herbal medicines during pregnancy and delivery. Major reasons for use were shortening of labor duration, 81 (38.9\%) and reducing labor pain, 58 (27.9\%). Independent predictors of herbal use were distance to the nearest health facility, adjusted prevalence ratio $[\mathrm{aPR}=1.12,95 \% \mathrm{Cl}=1.00,1.25]$, perception of safety about herbs, $[\mathrm{aPR}=1.16,95 \% \mathrm{Cl}=$ $1.05,1.29$ ] and health care providers stance on the use of herbs, [aPR $=1.14,95 \% \mathrm{Cl}=1.04,1.25]$.

Conclusion Use of herbal medicine during pregnancy and delivery in Tanzania is high. Researchers should consider designing comprehensive investigations on adverse effects of herbs to the mother and the fetus. Furthermore, health care providers ought to include health education messages during antenatal visits on the undesirable effects of using herbs.

\section{Plain English Summary}

In most of sub-Sahara African countries, herbal medicines are widely used during pregnancy and childbearing for various motives such ensuring positive pregnancy outcomes, easing labor and the general health of the woman and the baby in the womb. There are some studies which indicate that out of 10 women, about two to eight use herbs during pregnancy. The women and their babies in the womb may be at risk of the potentially poisonous materials contained in the herbs. There are some known effects of using herbal materials and these may even lead to the death of the mother or the baby in the womb or both. However, few data are available on the factors which increase the likelihood of pregnant women to use herbs.

In this study, we interviewed 342 mothers delivered a live baby in the preceding two years and attending Mother and Child Health clinics with their babies. Six out of 10 mothers reported having used herbal medicines during pregnancy or delivery. Findings show mothers who viewed herbal medicines as being safe when used in pregnancy or delivery, mothers discouraged by health care providers when attending antenatal clinic against using herbs as well as those who lived far away from a health facility tended to use herbs more. Studies like this provide evidence that there is need of having strategies to control use of herbal medicines in pregnancy. 


\section{Background}

It was recently estimated that maternal causes claimed 300000 lives of mothers annually and most were observed in Sub-Saharan countries [1]. In order to achieve the Sustainable Development Goal (SDG) of 70 maternal deaths per 100000 live births, efforts are being made to promote health facility delivery for all from about 60 percent being by traditional birth attendants. Traditional system exposures also include rampant use of herbal medicines which are habitually consumed raw [2]. In most of sub-Sahara African countries, herbal medicines are widely used during pregnancy and childbearing for various motives [3]. The drives include, for example, treating nausea and vomiting [4]; and strengthening pregnancy, positive pregnancy outcomes, ease labor and the general health of the woman and the fetus [5]. Frequently used herbal medicines by pregnant women in Tanzania include ginger (Zingier officinale), onions (Allium cepa) and Neem (Azadirachtaindica) [6]. In this study, herbal medicine use in pregnancy and delivery includes the utilization of plant products in their raw or cooked forms for various intentions as reported by the women themselves.

Despite their unclear pharmacology and potential toxicity, high proportions of women in Tanzania use herbal medicines during pregnancy and in inducing labor; 23\% in Mwanza [6]. A recent systematic review of literature [7] focusing on use of herbal medicines to induce labor by pregnant women revealed proportions of herbal use in the most recent pregnancy ranging from $6.5 \%$ in Ghana [8] to $80 \%$ in Uganda [9]. Some pregnant women often perceive herbal medicines as being natural and therefore safe, regardless of the unknown risks $[6,10]$.

Usually herbal medicines are taken towards the end of the gestation period or at the onset of labor pains, aimed to stimulate uterine muscles leading to stronger contractions and thus, hastening labor [9]. Certain herbs are believed to have the ability of inducing labor and shortening its duration [11]; alleviating labor pains, enhancing removal of a retained placenta, as well as toning the uterine muscles post delivery [6, 12].

Furthermore pregnant women in several parts of sub-Saharan Africa may use herbal remedies to treat pregnancy-related problems due to cost-effectiveness of therapy and easy access of these products [13]. Local herbs may be taken via the oral route in which they are most often consumed as a strong tea or chewed; rectal or vaginal routes and sometimes rubbing on the pregnant abdomen [14, 15]. Herbal remedies may also be used to protect the fetus from evil in-vitro [16] and to have a healthy child [17].

Nevertheless, the use of herbs during pregnancy should be a subject of concern as many common plants might have parts that contain natural toxins that could be dangerous if consumed. Additionally, when herbal medicines are taken in large amounts, they can be detrimental to pregnancy due to presence of some chemicals that can cross the placenta [18]. With the urgent need to achieve the SDGs, exposure to herbs has to be restricted to improve maternal health. Thus, this study aimed to assess the proportion of mothers using herbal medicines during pregnancy and delivery and to determine factors that are likely to predict the use in order to achieve the goal of improving maternal health. 


\section{Methods}

\section{Study design and study area}

The study adopted a cross-sectional analytical design involving women attending Reproductive, Maternal and Child Health (RMCH) clinics in a municipality setting of Tabora, in central Tanzania. According to the most recent national population census of 2012, Tabora Municipality has a total of 16709 women of childbearing age (15-49 years) [19]. Administratively, the municipality has two divisions; namely Tabora North and South with 14 and 15 wards respectively. The health system comprises of 44 health facilities of which 36 are public. Of the public health facilities, three are hospitals; three are health centers and 30 are dispensaries. Of all public health facilities, 34 provide RMCH services. The municipal report of 2012 shows that out of the 12020 women who attended antenatal care clinics, 10450 (96\%) delivered at health facilities [20].

\section{Sample Size Estimation And Sampling Procedure}

We estimated a sample of 342 women based on the formula $Z^{2} p(1-p) / m^{2}$; whereby $Z$ being a critical value of the normal distribution at $5 \%$ level of significance, $p$ is estimated percent $(23 \%)$ of pregnant women using herbal medicines [6] and $5 \%$ for $\mathrm{m}$, an estimated margin of error. We obtained a minimum sample of 273 that was adjusted for a possible $20 \%$ non-participation rate. The main inclusion criterion was a woman who delivered a live baby in the preceding two years.

RMCH services are provided by 18 and 16 health facilities in Tabora North and South divisions respectively. Proportionately, we estimated nine and eight health facilities respectively from Tabora North and South divisions. First, using systematic sampling technique, we selected health facilities from each of the two divisions. In the second stage, a random sampling technique was used to recruit women meeting the study criterion and attending RMCH clinics in the selected facilities on the days of data collection. Number selected in each facility was based on the proportion a facility contributed to maternal attendances in all selected facilities in a division put together in the previous month.

\section{Data Collection Procedure}

We collected data using semi- and structured interviews which elicited information on socio-demographic characteristics, use of herbs during the most recent pregnancy and delivery, perceived effects of using herbs in pregnancy, awareness of possible adverse effects as well as source of advice on the use of herbs. We assumed that all selected mothers were most likely to remember use of herbal medicines during their most recent pregnancy and delivery, thus, in position to give a self-report. The interview tool was first prepared in English and then translated into Kiswahili language, which is the medium of communication in Tanzania. In order to improve the content validity of the tool and other methodological issues as described by Castillo-Montoya [21] we pre-tested the instrument among few women in a near-by 
health facility that was not included in the study. Only mothers consenting were interviewed in a calm environment within the health facility at exit. A team of four research assistants trained on the research objectives and fieldwork procedures collected the data in September 2018, for a period of three weeks. The research assistants were registered nurses but not working in the respective clinic.

\section{Data Processing And Analysis}

We processed and analysed data using statistical software (Statistical Package for Social Sciences). Initially, by running the frequencies for all variables we were able to detect possible out-of-range values. We summarized categorical and quantitative variables using frequency tables. We performed bivariate analysis to assess the association between reported use of herbal medicines and selected independent variables using a Chi-square test. To assess independent predictors of obstetric herbal use, factors with $\mathrm{p}$ value $<0.2$ in the binary analyses were selected to enter into the Poisson regression model to predict use of herbs during the most recent pregnancy and delivery. Adjusted Prevalence Ratios (aPR) and their corresponding $95 \%$ confidence intervals are presented as both measures of association and their strengths. The significance level was set at $5 \%$.

\section{Results}

\section{Background characteristics of study participants}

We recruited 340women who delivered in the preceding two years (participation rate $99.4 \%$ ). The majority of the study participants, $142(41.8 \%)$, were in the age category of 26-35 years. Slightly more than half, 175 (51.5\%) reported completing primary education. Among all study participants, 285 (83.8\%) were married or cohabiting. Majority of the respondents, 177 (52.1\%) were either peasants or housewives whereas $159(48.5 \%)$ of their spouses were also peasants. Despite of $255(75.0 \%)$ mothers living within a walking distance of five $\mathrm{km}$ to the nearest health facility, only 141 (41.5\%) attended antenatal clinic (ANC) at least the four recommended visits. Most of the women, 246 (72.4\%), reported attending their first ANC when the pregnancy was at least three months (Table 1). 
Table 1

Distribution of respondents by their background characteristics $(n=340)$

\begin{tabular}{|ll|}
\hline Characteristic & Number (\%) \\
\hline Age group (years) & $83(24.4)$ \\
\hline $16-25$ & $142(41.8)$ \\
\hline $26-35$ & $115(33.8)$ \\
\hline $36+$ & \\
\hline Marital Status & $25(7.4)$ \\
\hline Never married & $285(83.8)$ \\
\hline Married/cohabiting & $30(8.8)$ \\
\hline Previously married & \\
\hline Education & $87(25.6)$ \\
\hline None/primary incomplete & $175(51.5)$ \\
\hline Primary complete & $78(22.9)$ \\
\hline Above primary & \\
\hline Woman's occupation & $177(52.0)$ \\
\hline Peasant/housewife & $127(37.4)$ \\
\hline Self-employed & $36(10.6)$ \\
\hline Formally employed & \\
\hline Occupation of spouse $(\mathrm{n}=328)$ & \\
\hline Peasant & $159(48.5)$ \\
\hline Self-employed & $246(72.7)$ \\
\hline Formally employed & \\
\hline Gestational age at booking & \\
\hline 0 - 3months & \\
\hline More than 3 months & \\
\hline Don't remember & \\
\hline Number of ANC visits & \\
\hline $4-J a n$ & \\
\hline
\end{tabular}




\begin{tabular}{|ll|}
\hline Characteristic & Number (\%) \\
\hline More than 4 & $141(41.5)$ \\
\hline Don't remember & $54(15.9)$ \\
\hline Distance to a nearest clinic $(\mathrm{Km})$ & \\
$\leq 5$ & $255(75.0)$ \\
$>5$ & $85(25.0)$ \\
\hline
\end{tabular}

\section{Herbal Use During The Most Recent Pregnancy And Delivery}

A large proportion of women, 208 (61.2\%), reported using herbal medicines during their most recent pregnancy and delivery. While the majority, $138(66.3 \%)$, reported high frequency use of herbal medicines (at least three times), 16 (7.7\%) and 54 (26.0\%) reported using herbal medicines either once or twice respectively. Of the 208 women who used herbal medicines, 57 (27.4\%) said herbal medicines were easily available and $83(39.9 \%)$ considered herbal medicines safe. The reported main routes of administration included oral, 135 (64.9\%), intra-vaginal, 59 (28.4\%), and topical application, $14(6.7 \%)$.

Of the 340 respondents, less than half, 164 (48.2\%) reported aware of adverse effects of using herbal medicines in pregnancy and delivery. Surprisingly, significantly more women, 153 (93.3\%), reported aware of the effects of using herbal medicines during pregnancy and delivery as compared to $55(31.3 \%)$ who were not aware $(p<0.01)$. Regardless of herbal use status, $70(20.6 \%)$ mentioned uterine rupture, 51 (15.0\%) excessive vaginal bleeding, 22 (6.5\%) cited uterine infection and $21(6.2 \%)$ reported a possibility of death of the mother or baby as untoward consequences of using herbs in pregnancy.

Among 208 mothers who ascertained having used herbs during their most recent pregnancy and delivery, 81 (38.9\%) thought the most benefits of using herbal medicines were shortening of duration of labor and $58(27.9 \%)$ alleviating labor pains (Fig. 1).

\section{Determinants of the use of herbal medicines in the most recent pregnancy and delivery}

In Table 2, we present results of bivariate analysis between use of herbal medicine during the most recent pregnancy and selected independent variables. Factors that were significantly associated with herbal medicine use included living far from a health facility, perceiving herbs as safe, source of advice towards using herbs and receiving advice against using herbs from health care providers. 
Table 2

Association of herbal use in the most recent delivery and selected characteristics

\begin{tabular}{|c|c|c|}
\hline \multirow[t]{2}{*}{ Characteristic } & Herbal use & Chi-square, p-value \\
\hline & Number (\%) & \\
\hline Age group (years) & & $1.68,0.431$ \\
\hline $16-25$ & $46(55.4)$ & \\
\hline $26-35$ & $88(62.0)$ & \\
\hline $36+$ & $74(64.3)$ & \\
\hline Education level & & $2.85,0.239$ \\
\hline None/prim incomplete & $50(57.5)$ & \\
\hline Primary complete & $104(59.4)$ & \\
\hline Secondary and above & $54(69.2)$ & \\
\hline Occupation of spouse $(\mathrm{N}=316)$ & & $4.59,0.101$ \\
\hline Peasant & $103(64.8)$ & \\
\hline Formerly employed & $40(51.3)$ & \\
\hline Self-employed & $59(64.8)$ & \\
\hline Distance to nearest facility (km) & & $5.35,0.014$ \\
\hline$\leq 5$ & $147(57.6)$ & \\
\hline$>5$ & $61(71.4)$ & \\
\hline Perceived availability of herbs & & $1.51,1.139$ \\
\hline Easily available & $39(68.4)$ & \\
\hline Not easily available & $169(59.7)$ & \\
\hline Perceived safety of herbs ( $N=321)$ & & $11.73,<0.01$ \\
\hline Safe & $64(77.1)$ & \\
\hline Not safe & $144(56.0)$ & \\
\hline Source of advice & & $42.02,<0.01$ \\
\hline Friends & $65(89.0)$ & \\
\hline Parents & $33(40.2)$ & \\
\hline
\end{tabular}




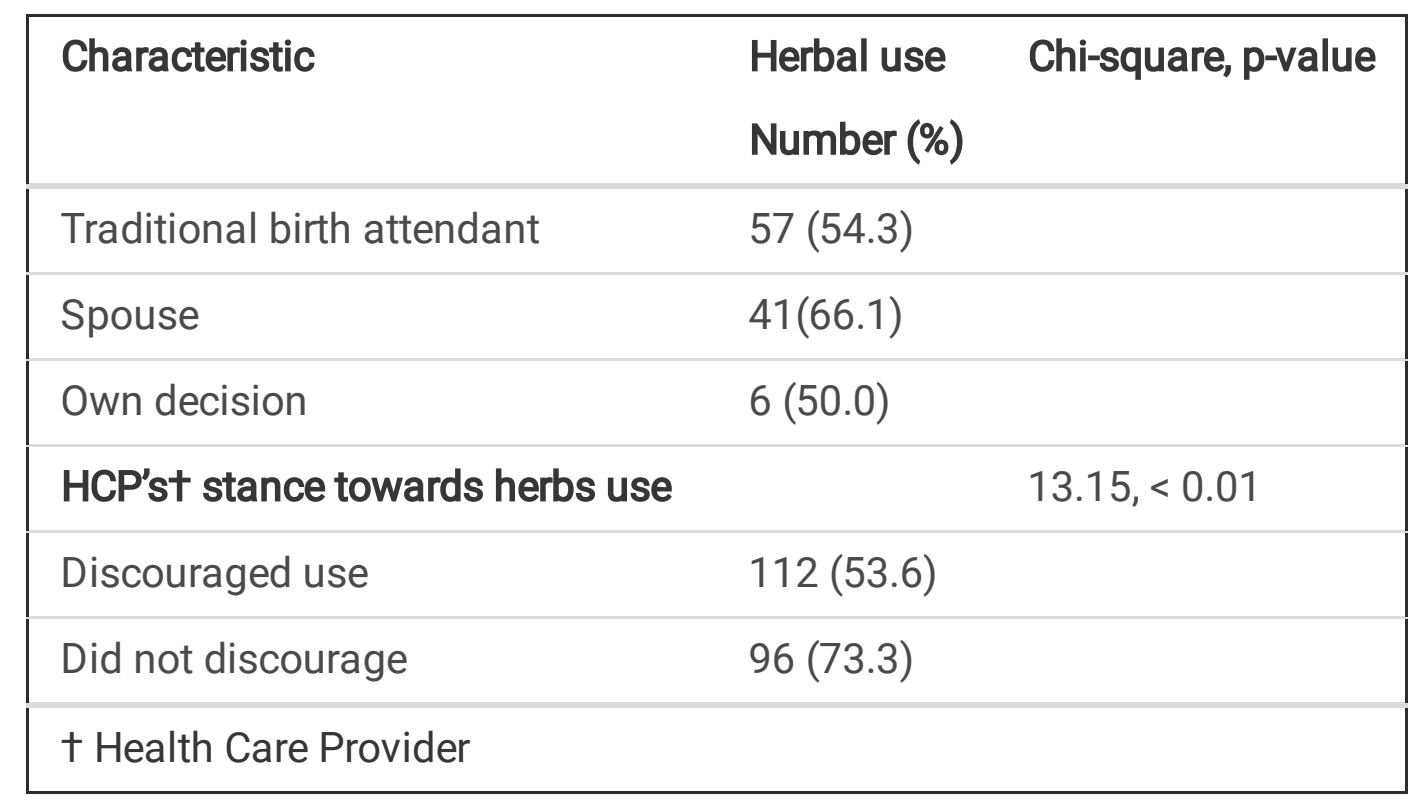

In Table 3, we display predictors of herbal use during the most recent pregnancy and delivery among mothers who attended reproductive and child health clinics. Predictors of herbal use were distance to the nearest health facility, women's perception of herbal safety and whether the health care provider discouraged use of herbal medicines or not during antenatal visits. For example, mothers who perceived herbal medicines as being safe had $16 \%$ adjusted prevalence ratio to use herbal medicines, [aPR $=1.16$, $95 \% \mathrm{Cl}=1.05,1.29]$ as compared to those perceiving herbal medicines being unsafe. Furthermore, mothers confirming that during antenatal care visiting, health care providers did not discourage use of herbal medicines had almost $15 \%$ higher prevalence ratio of herbal medicine use as compared to those saying that health care providers discouraged the use of herbal medicine, [aPR $=1.14,95 \% \mathrm{Cl}=1.04,1.25]$. 
Table 3

Correlates of herbal use during the most recent pregnancy and delivery among women in Tabora municipality

\begin{tabular}{|c|c|c|c|}
\hline & Herbal use & aPR $(95 \% \mathrm{Cl}) \star$ & $\mathrm{p}$-value \\
\hline Factor & Number (\%) & & \\
\hline \multicolumn{4}{|l|}{ Occupation of spouse } \\
\hline Peasant & $103(64.8)$ & $1.05(0.92,1.20)$ & 0.455 \\
\hline Formal employment & $40(51.3)$ & $1.12(0.96,1.30)$ & 0.143 \\
\hline Self-employment & $59(64.8)$ & Reference & \\
\hline \multicolumn{4}{|c|}{ Distance to nearest health facility (km) } \\
\hline \multicolumn{4}{|l|}{$\leq 5$} \\
\hline$>5$ & $147(57.6)$ & $1.12(1.00,1.25)$ & 0.049 \\
\hline Perceived safety of herbs & $61(71.4)$ & Reference & \\
\hline \multicolumn{4}{|l|}{ Very safe } \\
\hline Not safe & $64(77.1)$ & $1.16(1.05,1.29)$ & 0.004 \\
\hline HCP'st stance towards herbs use & $144(56.0)$ & Refernce & \\
\hline Did not discourage use of herbs & $112(53.6)$ & $1.14(1.04,1.25)$ & 0.002 \\
\hline Discouraged use & $96(73.3)$ & Reference & \\
\hline
\end{tabular}

\section{Discussion}

In this study, we assessed use of herbal medicines and their determinants among mothers who delivered in the preceding two years and who were attending RMCH clinics in health facility settings in Tanzania. The findings show that more than $60 \%$ of the women used herbs during the most recent pregnancy or delivery. The significant predictors of use were distance to the nearest health facility, women's perception of herbal safety and whether the health care provider discouraged use of herbal medicines or not during antenatal visits.

The results of the present study ranges between $20-80 \%$ as reported in other related studies $[6,9,13,21$, 22]. Furthermore, these findings are in agreement with other studies elsewhere, which confirm dramatic increment in the use of herbal medicines in pregnant women. The possible reason for the observed high usage of herbs could be due to easy availability, their perceived safety and rapid increase in promotion of 
traditional medicines in the society as well as the media. It is common to see posters advertising traditional medicines and herbalists in various parts Tanzania.

A significant association has been documented between educational status of a woman and utilization of herbal remedies during pregnancy and delivery. For example, some studies in Africa suggest that women of low educational status as compared to those highly educated had a higher likelihood of using herbal remedies $[13,21,22]$. However, the current data do not support such an association. In addition, the education status of the mother did not have significant influence on the use of herbs in the most recent pregnancy or during delivery.

Plants used for herbal remedies are less expensive as compared to modern medicines and they have been culturally considered as an effective and acceptable option even when modern health facilities are available [24]. Some studies have associated use of herbs during pregnancy with lack of access to public health care [7]. Similar to observations by Mothupi, use of herbal medicines in this study is significantly associated with perceived beliefs about their safety and local availability [10].

In our study, women who resided far away (at least $5 \mathrm{~km}$ ) to the nearest health facility had more than $10 \%$ adjusted prevalence ratio to use herbal medicines compared to women living closer to the health facilities. This may suggest that accessibility to public health care is an important attribute to using herbs during pregnancy, similar to observations elsewhere $[3,7,13]$.

Medications and in particular herbs should be used with caution during pregnancy as could result in adverse effects. Apart from not having standard dosages, the pharmacology and potential toxicity of the plants used are still unclear [7]. The most important aspect is lack of awareness of pregnant women and the community about potential effects of using herbal medicines on the mother and the fetus. In the current study, less than half (48\%) of the respondents were aware of the adverse effects of herbs in pregnancy and only a few of them were able to mention some of the major effects. In Ethiopia and Norway, only $14 \%$ and $12 \%$ respectively of pregnant women reported to have received health advice from healthcare workers on the use of herbs [22, 24]. Compared to women who were discouraged by health care providers against the use of herbs during pregnancy, significantly more women in the Tabora study who did not receive such advice used herbs $(p<0.01)$. This finding suggests that if women get appropriate information during pregnancy they will greatly avoid using the herbs.

Similar to studies in Ghana [14] and Ethiopia [13], oral route was the commonest means (64.9\%) for taking herbs during pregnancy and labor in the Tabora study. In all these studies, women most often chewed or consumed herbs as a strong tea. On the contrary, findings in a related study in Kagera regional referral hospital, in rural north-western Tanzania revealed that only $22 \%$ of women reported using herbs orally [15]. However, the Kagera study looked at only women who used herbs for inducing abortion.

The main motive for using herbs in the current study was to shorten labor duration (38.9\%). This finding is consistent with what was observed in studies in Ghana and Mwanza Tanzania, which revealed that $39 \%$ and $68 \%$ of women respectively took herbal medicines for shortening duration of labor and alleviate 
pains [6]. For many years, women used herbal medicines in pregnancy to help several conditions during delivery process. For example, Nigerian women use herbal medicine to prevent complications, such as pain and bleeding [12]. Similar to findings in Tabora, studies indicate that pregnant women in some communities use herbs for the purpose of accelerating labor, preventing antepartum and postpartum haemorrahage, increasing milk production, and aiding postpartum uterine involution $[6,12]$. The grounds for the high usage of herbs could in addition be attributed to their easy accessibility, perceptions that they are safe and alleviate pain; as well as the general lack of awareness of the potential side effects $[13,25]$.

In the Tabora study, independent predictors of using herbs during the most recent pregnancy are distance to the nearest facility, perceived safety of the herbs and the stance of health care providers towards use of the herbs. The statistically significant association between perception of safety and use of herbal remedy during pregnancy is in line with the findings of various related studies elsewhere $[4,21,26]$. The studies indicate that women who perceive herbal remedies as safe use them more than those who have negative perception about the herbs.

This study has several potential limitations. First, although we assumed mothers gave birth within two years prior to the survey were likely to remember use of herbal medicines in their most recent pregnancy; we are unable to rule completely out the possibility of information bias due to individual's ability to recall. If some of women were unable to remember, this bias could have contributed to the low estimates. Second, women were aware that use of herbal medicine during pregnancy was discouraged at each health facility. Therefore, due to social desirability bias, there is a possibility that some women concealed reporting use of herbal medicines, thus, leading to under-estimating the proportion of herbal use. Third, no research design is optimal for all purposes and since this was a cross-sectional study, it might have reservations with the generalizability of the findings. Fourth, there is possibility that the variables we considered as independent may not be exhaustive.

\section{Conclusions}

The results of the present study point out that as high as $60 \%$ of women possibly in most municipal settings in Tanzania use herbs for various reasons during pregnancy and labor. Perceived safety of the herbs and health care providers' stance towards use of herbs as well as physical distance to the nearest facility were significant predictors of the use of herbs. The study forms basis for designing intervention programs to address use of herbs during pregnancy and underscores the important role of health care providers attending pregnant women in the antenatal clinics. This is important given the high prevalence of the use of herbs during pregnancy and delivery in our community, and the relative lack of evidence of safety. Therefore, first, we recommend that researchers consider designing comprehensive investigations on adverse effects of herbal medicines used in pregnancy on the mother and the fetus. Second, health care providers ought to include health education messages on the undesirable effects of using herbs in the routine sessions they provide to women when attending antenatal care services.

\section{Abbreviations}


ANC

Antenatal Care; aPR:Adjusted Prevalence Ratio; Cl:Confidence Interval; HCP:Health Care Provider; PR:Prevalence Ratio; RMCH:Reproductive, Maternal and Child Health; SDGs:Sustainable Development Goals.

\section{Declarations}

\section{Ethics approval and consent to participate}

The Muhimbili University of Health and Allied Sciences (MUHAS) Institutional Review Board approved the protocol. The Tabora Municipal Executive Director and the Municipal Medical Officer granted permission to conduct the study in the selected health facilities. We explained the aims and benefits of the study to each selected participant. We emphasized voluntary participation such that each participant was free to respond or not, to each question and to stop responding to any question at any point regardless of the services she was expecting to get. We assured strict anonymity and confidentiality to all individual participants. Before the interviews, each potential survey respondent was asked to sign (or thumb-print) the consent form. Furthermore, no names were recorded on the survey tool.

\section{Consent for publication}

Not applicable since no individual level data is shown.

\section{Availability of data and materials}

All data generated or analyzed during this study are included in this article and are available from the corresponding author on request as per institutional guidelines.

\section{Competing interests}

The authors declare no competing interests

\section{Funding}

Data collection was supported by the CDC-School of Public Health and Social Sciences project, Muhimbili University of Health and Allied Sciences. The funders had no role in the study neither in the decision to publish.

\section{Authors' contributions}

ATK and GCM conceptualized and developed the protocol. ATK guided data collection while GCM supervised field activities. ATK and GCM analyzed and interpreted the data and wrote the manuscript. All authors read and approved the final manuscript.

\section{Acknowledgements}


We are thankful to the Tabora Municipal Executive Director and Medical Officer of Health for allowing us to conduct the study in their area of jurisdiction. We express gratitude to all the mothers who volunteered their time and information. We also extend our appreciations to the research assistants for collecting the data.

\section{References}

1. United Nations. The Sustainable Development Goals Report [Internet]. 2019 [cited 2020 Apr 20]. Available from: https://unstats.un.org/sdgs/report/2019/The-Sustainable-Development-GoalsReport-2019.pdf.

2. Nikolajsen T, Nielsen F, Rasch V, Sørensen PH, Ismail F, Kristiansen U, et al. Uterine contraction induced by Tanzanian plants used to induce abortion. J Ethnopharmacol. 2011 Sep;137(1)(1):9215.

3. Nyeko R, Tumwesigye NM, Halage AA. Prevalence and factors associated with use of herbal medicines during pregnancy among women attending postnatal clinics in Gulu district, Northern Uganda. BMC Pregnancy Childbirth. 2016 Oct 6;16(1).

4. Hollyer $T$, Boon $H$, Georgousis A, Smith $M$, Einarson $A$. The use of CAM by women suffering from nausea and vomiting during pregnancy. BMC Complement Altern Med. 2002;2.

5. Chakona G, Shackleton C. Food taboos and cultural beliefs influence food choice and dietary preferences among pregnant women in the eastern Cape, South Africa. Nutrients [Internet]. 2019 Nov 1 [cited 2020 Apr 8];11(11). Available from: http://www.ncbi.nlm.nih.gov/pubmed/31694181.

6. Dika H, Dismas M, Iddi S, Rumanyika R. Prevalent use of herbs for reduction of labour duration in Mwanza, Tanzania: Are obstetricians aware? Tanzan J Health Res. 2017;19(2):1-8.

7. Ngoma CM. Use of Herbal Medicines to Induce Labour by Pregnant Women: A Systematic Review of Literature. JOJ Nurs Heal Care. 2017;2(3):7-12.

8. Adusi-Poku Y, Vanotoo L, Detoh E, Oduro J, Nsiah R, Natogmah A. Type of herbal medicines utilized by pregnant women attending ante-natal clinic in Offinso north district: Are orthodox prescribers aware? Ghana Med J. 2015 Mar 2;49(4):227-32.

9. Kamatenesi-Mugisha M, Oryem-Origa $\mathrm{H}$. Medicinal plants used to induce labour during childbirth in western Uganda. J Ethnopharmacol. 2007;109(1):1-9.

10. Mothupi MC. Use of herbal medicine during pregnancy among women with access to public healthcare in Nairobi, Kenya: A cross-sectional survey. BMC Complement Altern Med. 2014;14(1):18.

11. Panganai T, Shumba P. The African Pitocin - A midwife's dilemma: The perception of women on the use of herbs in pregnancy and labour in Zimbabwe, Gweru. Pan Afr Med J. 2016 Sep 19;25(5).

12. Attah AF, O'Brien M, Koehbach J, Sonibare MA, Moody JO, Smith TJ, et al. Uterine contractility of plants used to facilitate childbirth in Nigerian ethnomedicine. J Ethnopharmacol. 2012 Aug;30(1):377-82. 143(. 
13. Laelago T, Yohannes T, Lemango F. Prevalence of herbal medicine use and associated factors among pregnant women attending antenatal care at public health facilities in Hossana Town, Southern Ethiopia: Facility based cross sectional study. Arch Public Heal. 2016;74(1).

14. Addo VN. Herbal Medicines: Socio-Demographic Characteristics And Pattern Of Use By Patients In A Tertiary Obstetrics And Gynaecology Unit. J Sci Technol. 2007;27(3):149-55.

15. 10.1186/s12884-014-0419-6 Rasch V, Sørensen PH, Wang AR, Tibazarwa F, Jger AK. Unsafe abortion in rural Tanzania - The use of traditional medicine from a patient and a provider perspective. BMC Pregnancy Childbirth [Internet]. 2014 Dec 1 [cited 2020 Apr 1];14(1):419. Available from: http://bmcpregnancychildbirth.biomedcentral.com/articles/10.1186/s12884-014-0419-6.

16. Maputle S, Khoza L, Lebese R. Knowledge towards Pregnancy-induced Hypertension among Pregnant Women in Vhembe District, Limpopo Province. J Hum Ecol. 2015 Jul;51(1-2):47-54.

17. Nalumansi PA, Kamatenesi-Mugisha M, Anywar G. Medicinal Plants used during Antenatal Care by Pregnant Women in Eastern Uganda. Afr J Reprod Health [Internet]. 2017 Dec [cited 2020 Apr 24];21(4):33-44. Available from: http://www.ncbi.nlm.nih.gov/pubmed/29624949.

18. Maliwichi-Nyirenda CP, Maliwichi LL. Medicinal plants used for contraception and pregnancy-related cases in Malawi: A case study of Mulanje District. J Med Plants Res. 2010;4(24).

19. NBS. Population and Housing Census: Population Distribution by Administrative Areas. National Bureau of Statistics; 2012.

20. RMCH. Tabora Municipal Reproductive, Maternal, Newborn and Child Health Report. 2012.

21. Castillo-Montoya M. Preparing for Interview Research: The Interview Protocol Refinement Framework [Internet]. 2016. Available from: https://nsuworks.nova.edu/tqr/vol21/iss5/2.

22. Olowokere $A E$, Olajide 0 . Women's perception of safety and utilization of herbal remedies during pregnancy in a local government area in Nigeria. Clin Nurs Stud. 2013;1(4):9-22.

23. Bayisa B, Tatiparthi R, Mulisa E. Use of herbal medicine among pregnant women on Antenatal care at Nekemte Hospital, Western Ethiopia. Jundishapur J Nat Pharm Prod. 2014;9(4):4-8.

24. Chikezie PC. Herbal Medicine: Yesterday, Today and Tomorrow. Altern Integr Med. 2015;4(3).

25. Nordeng H, Havnen GC. Use of drugs in pregnancy: A survey among 400 Norwegian Women. Pharmacoepidemiol Drug Saf. 2004;13(6):371-80.

26. John LJ, Shantakumari N. Herbal medicines use during pregnancy: A review from the middle east. Oman Med J. 2015;30(4):229-36.

27. Mohamad TAST, Islahudin F, Jasamai M, Jamal JA. Preference, perception and predictors of herbal medicine use among malay women in Malaysia. Patient Prefer Adherence. 2019;13:1829-37.

\section{Figures}




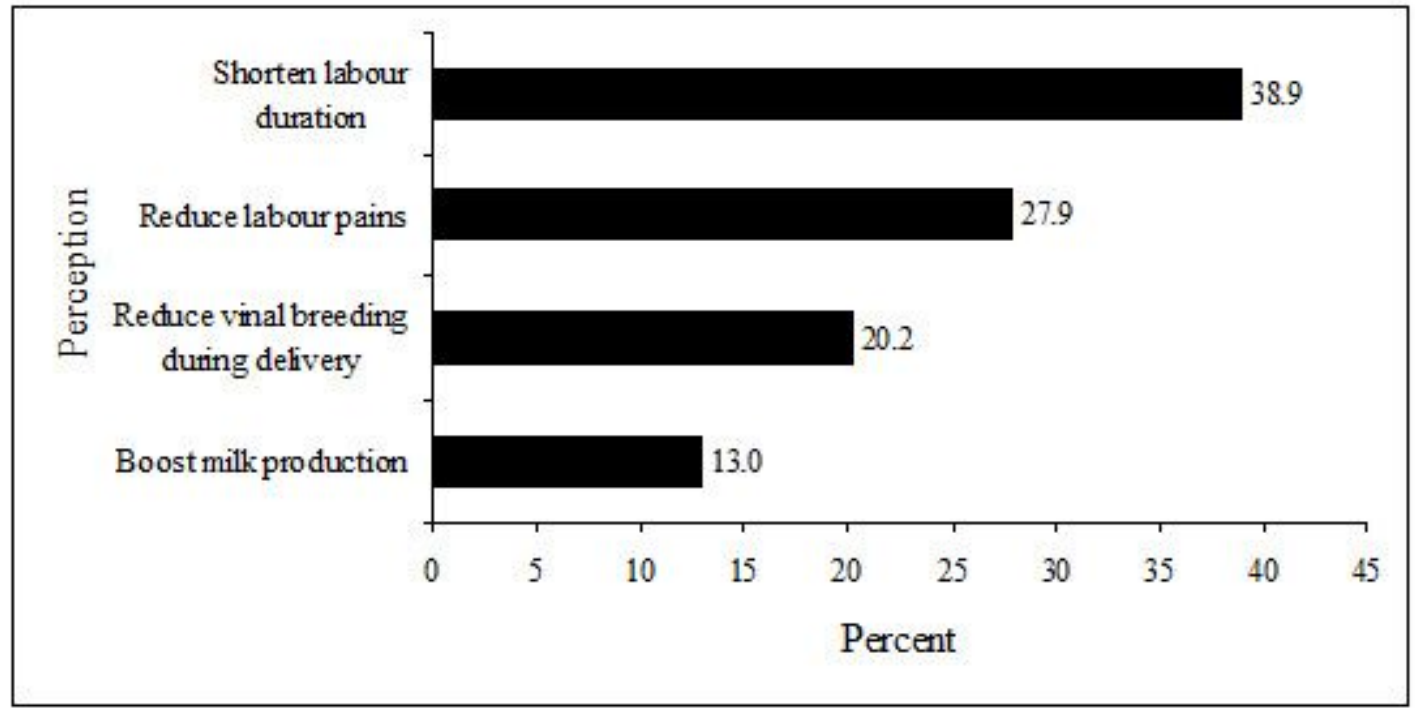

Figure 1

Most perceived benefits of using herbal medicines in pregnancy and labor 